\title{
Ventilator Associated Pneumonia (VAP) in Neonatal Intensive Care Unit - An Emerging Problem
}

\author{
Nandini Vijayakanthi • Srinivasan Kitchanan • \\ Durai Arasan
}

Received: 14 June 2013 / Accepted: 12 August 2014 / Published online: 4 September 2014

(C) Dr. K C Chaudhuri Foundation 2014

To the Editor: Ventilator Associated Pneumonia (VAP), especially with multiresistant organisms has been increasing in the Neonatal Intensive Care Units (NICU) [1-5]. There are few published data from India.

In this retrospective observational study, medical case records of neonates who required mechanical ventilation for $>48 \mathrm{~h}$ were evaluated to determine the incidence, risk factors, morbidity and mortality of VAP in the NICU of Institute of Child Health, Chennai, India between January 1 and October 31, 2007. VAP was diagnosed using the Center for Disease Control and Prevention (CDC) criteria for diagnosis of clinically defined nosocomial pneumonia in infants. The criteria includes: Worsening gas exchange, increasing oxygen requirements, or increasing ventilator settings and at least three of the following: temperature instability; leukopenia or leukocytosis and left shift; increasing respiratory secretions or increasing suctioning requirements; apnea, tachypnea, nasal flaring with retraction of chest wall or grunting; wheezing, rales, or rhonchi; bradycardia or tachycardia and two or more serial chest radiographs with at least one of the following: new or progressive and persistent infiltrate; consolidation; cavitation; pneumatoceles.

Of the 265 mechanically ventilated neonates enrolled in the study, 135 neonates entered the study cohort. The incidence of VAP was 22.22 cases per 100 mechanically ventilated neonates. Klebsiella $(66.67 \%)$ was the predominant organism isolated from the lower respiratory tract specimen (LRT) collected through the endotracheal tube. Home delivery, respiratory distress at admission, unstable cardiopulmonary assessment at admission defined as atleast one of the following: unstable airway/abnormal breathing/abnormal circulation/altered mental

\footnotetext{
N. Vijayakanthi $(\bowtie) \cdot$ S. Kitchanan $\cdot$ D. Arasan Division of Neonatology, Institute of Child Health \& Hospital for Children (ICH\& HC), Madras Medical College, Egmore,

Chennai 600 008, India

e-mail: nandini_mmc@yahoo.co.in
}

status, repeated intubations (more than 1), prolonged ventilation, prolonged duration of hospitalization and level III stay were found to significant risk factors for VAP by univariate analysis. Factors that retained significance in multivariate logistic regression model were unstable initial cardio pulmonary assessment ( $p$ value $=0.010$, adjusted OR: $0.2,95 \%$ CI: $0.0,0.6)$ and repeated intubations ( $p$ value, 0.001 , adjusted OR: $34.3,95 \% \mathrm{CI}$ : $8.3,142.4)$. The mortality rates for the neonates with VAP was $50 \%$ and for those without VAP was $69.5 \%$ ( $p$ value $=0.030$ ).

VAP is a serious nosocomial infection. Preventable risk factors should be addressed in all neonatal units. Further research is necessary to formulate the guidelines for diagnosis of VAP in neonates.

Acknowledgments The authors thank Dr. Githa K and Dr. Rema Chandramohan, Division of Neonatology for their support and guidance. They also acknowledge the support of Mr. Chinnayan Ponnraja, ICMR, Chennai for his assistance in the statistics of the study.

Conflict of Interest None.

Source of Funding None.

\section{References}

1. Goldsmith JP, Edward HK. In: Assisted ventilation of the neonate. Chapter 24, 5th ed. Saunders: Elsevier; 2011. p. 426-35.

2. McGrath EJ, Asmar BI. Nosocomial infections and multidrug-resistant bacterial organisms in the pediatric intensive care unit. Indian J Pediatr. 2011;78:176-84

3. Apisarnthanarak A, Holzmann-Pazgal G, Hamvas A, Olsen MA, Fraser VJ. Ventilator associated pneumonia in extremely preterm neonates in a neonatal intensive care unit: characteristrics, risk factors, and outcomes. Pediatrics. 2003;112:1283-9.

4. Petdachai W. Ventilator-associated pneumonia in a new born intensive care unit. Southeast Asian J Trop Med Public Health. 2004;35:724-9.

5. Foglia E, Meier MD, Edward A. Ventilator-associated pneumonia in neonatal and pediatric intensive care unit patients. Clin Microbiol Rev. 2007;20:409-25. 